\title{
Feasibility analysis of leaf disc samples produced via agroinfiltration for promoter trapping studies
}

\author{
D. Natorajan ${ }^{1,3}$, H.Y. Yong ${ }^{1,3}$, I. Ismail ${ }^{1,2 *}$ and Z. Zainal ${ }^{1,2}$ \\ ${ }^{1}$ School of Biosciences and Biotechnology, Faculty of Science and Technology, Universiti \\ Kebangsaan Malaysia, Bangi, 43600 Selangor, Malaysia; ${ }^{2}$ Centre for Plant Biotechnology, \\ Institute of System Biology, Universiti Kebangsaan Malaysia, Bangi, 43600 Selangor, \\ Malaysia; ${ }^{3}$ Current address: ACGT Sdn Bhd, Technology Park Malaysia, \\ Bukit Jalil, 5700, Kuala Lumpur, Malaysia
}

\begin{abstract}
Promoter trapping is a method used to isolate and characterize regulatory regions from genomes by elucidating the expression of a promoterless reporter gene flanked by two transposable elements. The conventional method of promoter trapping requires the generation of stable transformed tissue culture derived plant lines. Nevertheless,this method can be laborious, time consuming and expensive. As an alternative method, leaf disc samples produced via leaf agroinfiltration was employed in this work. A promoter trapping construct named pCAMDIN was created which contains a promoterless GUS ( $\beta$-glucuronidase) gene flanked by the left and right T-DNA border using pCAMBIA 1301 and pBI 121. Following that, the protocol for agroinfiltration of tomato plants using both direct agroinfiltration and vacuum agroinfiltration was optimized. Non-destructive protocols for detection of GUS genes were tested and optimised. Following that, GUS gene expression was studied and areas which showed expression were isolated by making leaf punches. Analysis was carried out by Southern blotting and T-DNA fingerprinting to determine the gene copy number.
\end{abstract}

Keywords: Agrobacterium tumefaciens, T-DNA insertion, agroinfiltration, non-destructive GUS detection

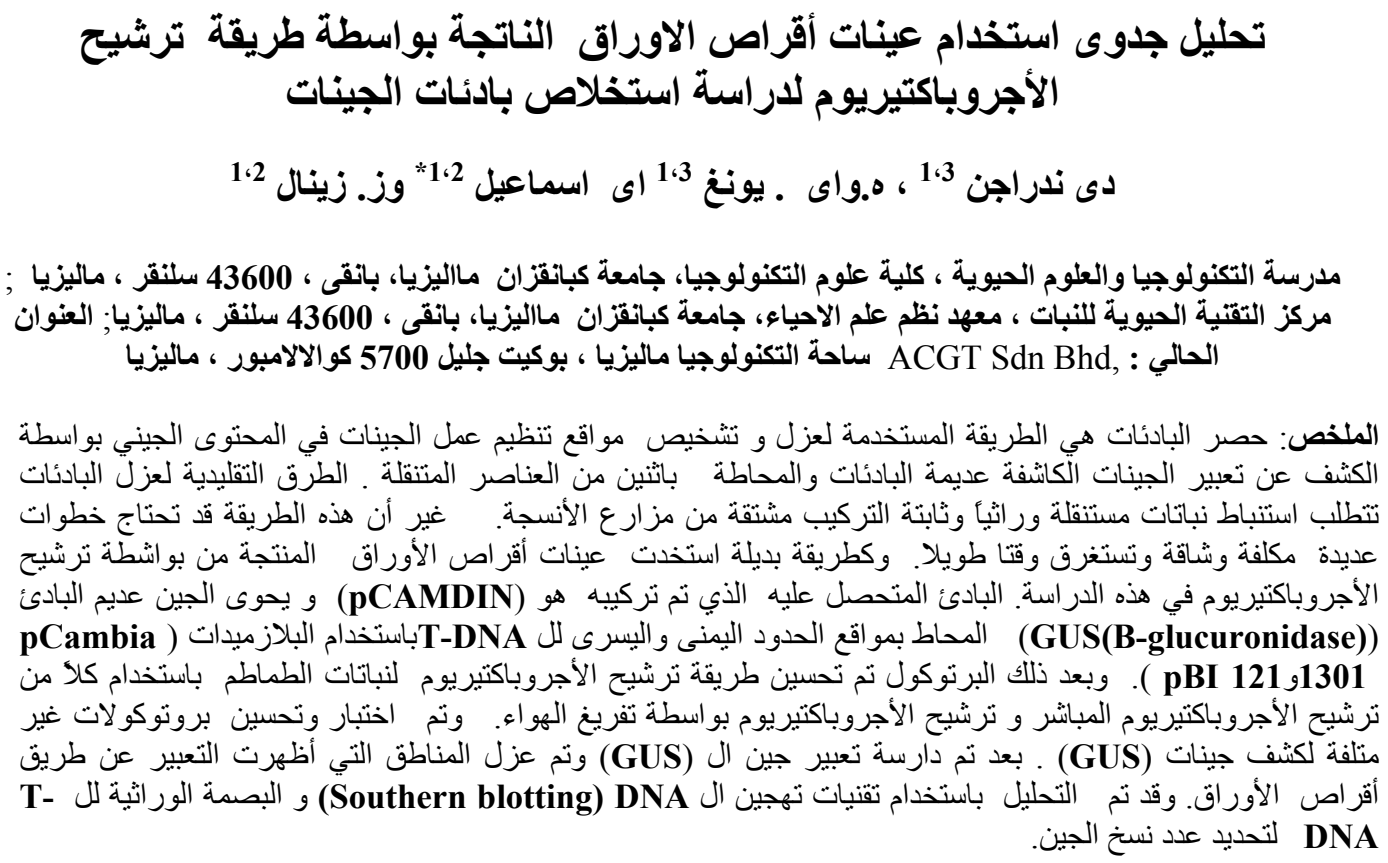

*Corresponding Author, Email: maniz@ukm.my 


\section{Introduction}

Promoters control the fundamental pattern of gene expression in an organism and thus are of great interest for understanding plant gene expression patterns leading to wide interest in their uses (Yang et al., 2004). Genetic modification in plants requires tissuespecific promoters to achieve targeted expression of the gene of interest in order to avoid the problem of undesired ectopic gene expression caused by using constitutive promoters (Anuradha et al., 2006). In order to study the functional genomics of plants, insertional mutagenesis is used as a powerful tool which allows for genome-wide identification and isolation of functionally redundant genes. A procedure not feasible when using conventional mutagenesis approaches (Serge et al., 2005). Insertional mutagenesis to isolate and characterize plant regulatory sequences can be done via ethylmethanesulfonate (EMS), fastneutron treatment and transposable elements such as T-DNA (Walden, 2002). An extensive volume of work exists in Arabidopsis thaliana for creating insertional mutant pools to enable functional genomics analysis (Parinov and Sundaresan, 2000; Bouche' and Bouchez, 2001; Sessions et al., 2002; Szabados et al., 2002) and a rice mutant library containing 55,000 lines is available for research (Hsing et al., 2007). All of the methods for making the insertional mutation pools discussed above rely on creating stable transformed plant lines which carries at least one T-DNA insertion (Galbiati et al., 2000). The process of creating these lines require tissue culture methods in order to propagate and develop the plants to the stage where they can be grown in greenhouses and this can be very costly, time consuming and laborious (Sreenivasan and Saha, 2009).

Promoter trapping is a method used to detect the presence of promoter sequences within genomic regions where T-DNA is used to insert mobile elements containing a promoterless reporter gene flanked by transposable elements into the plant genome (Sundaresan et al., 1995). In plants this method is commonly used in conjunction with T-DNA transfer of Agrobacterium sp. and consequent regeneration of transformed lines from stable transformants. Promoter trapping allows for the elucidation of genes which are specifically expressed in restricted cell types or are temporally modulated (Abuin et al., 2007; Sreenivasan and Saha, 2009).

Analysis of GUS expression will enable the detection, isolation and sequencing of the promoter region from the upstream location of the T-DNA integration. One of the main hurdles in this process is that it requires a large amount of stable non-chimeric transformants which needs to be generated into transformed lines via tissue culture methods in order to conduct further studies of the T-DNA integration event. The problem raised in cases where the plant of interest is considered as 'tissue culture recalcitrant'. One potential solution is to generate multiple insertions by directly infiltrating the stroma of the plant leaves with Agrobacterium tumefaciens carrying the promoterless GUS plasmid, followed by analysis of the integration event in areas showing expression.

This paper proposes a method that can be used to study insertional mutagenesis using T-DNA for the elucidation of regulatory elements in a larger scale and at a faster rate by omitting the requirements for the generation of promoter tagging plant lines. The method uses direct leaf agroinfiltration to generate leaf disc samples for promoter trapping studies which will enable the isolation and characterization of promoters from plant genomes. Understanding these promoters will provide many insights into plant gene expression as well as providing tools that can be used for genetic engineering of 
plants in the future. Genetic engineering of plants will require multiple promoters with different expression specificity, functions and sequence similarity to allow for the stacking of multiple genes.

\section{Materials and Methods}

\section{Construction of the promoterless tagging plasmid pCAMDIN}

For T-DNA tagging, a promoterless plasmid construct was designed from pCAMBIA 1301 and pBI 121. Using the restriction enzymes EcoRI \& BamHI, a promoterless gus gene was excised from pBI 121 and the gus gene with promoter was removed from pCAMBIA 1301. Using ligation with T4 DNA Ligase, the promoterless gus gene was inserted into pCAMBIA 1301 in the desired orientation. Antibiotic selection, enzymatic digestion, PCR and plasmid sequencing were carried out to confirm the identity, orientation of insertion and the location of insertion in the new plasmid which was subsequently named pCAMDIN (Figure 1). The plasmid pCAMDIN was inserted into the Eschericia coli XL-1 Blue for propagation and then inserted into Agrobacterium tumefaciens for plant transformation work.

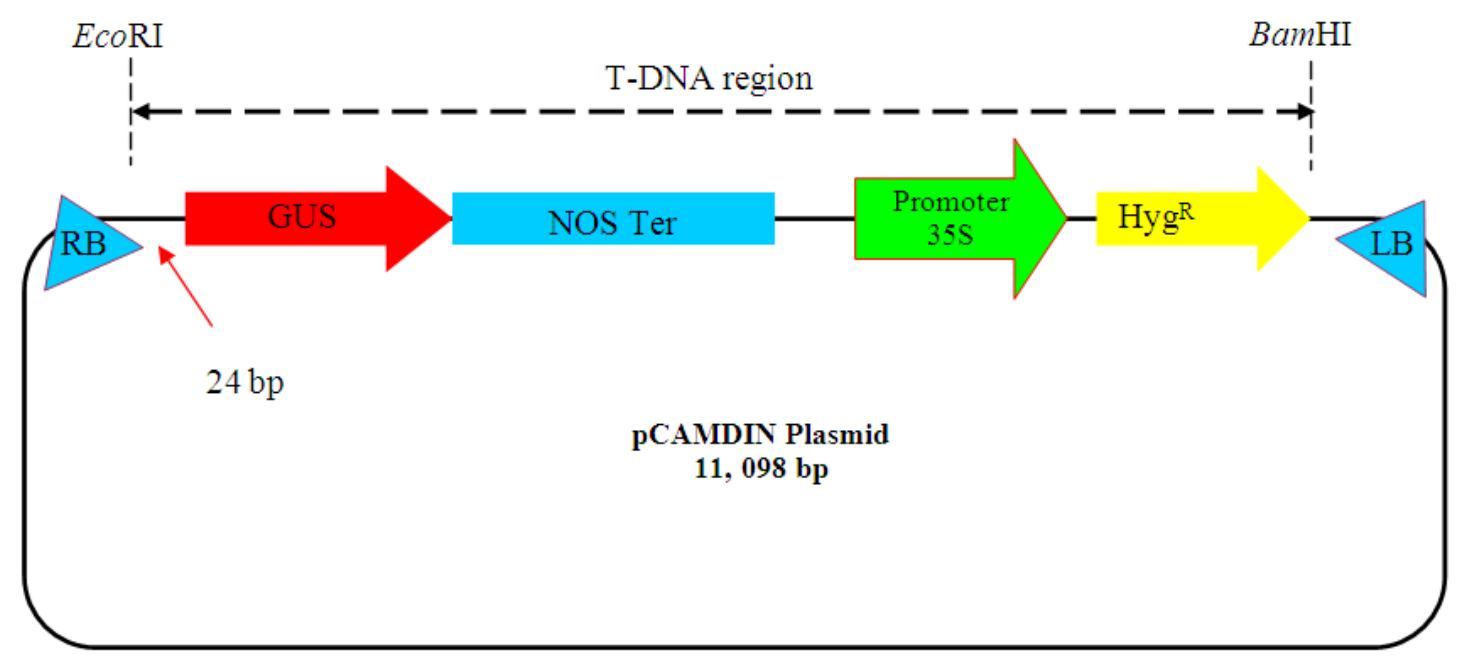

Figure 1. Schematic diagram of pCAMDIN plasmid.

\section{Agroinfiltration}

Agroinfiltration is a process to directly insert $A$. tumefaciens cells carrying the desired transgene into the plant leaf stoma via a syringe without needle which is placed on the leaf surface and then pressure is applied gently to force the solution containing A. tumefaciens into the leaf. Optimization had been carried out to determine the best buffer solution, acetosyringone concentration and agroinfiltration treatment. Methods as used by Van der Hoorn et al. (2000) was adopted and modified to identify the best protocol for agroinfiltration of tomatoes (Results not published). Based on our experimentations the optimal protocols for tomato leaf agroinfiltration are as follows. Fresh A. tumefaciens culture is grown to an optical density reading of 0.6 at $600 \mathrm{~nm}$ and was pelleted by centrifugation, then 20 $\mu \mathrm{M}$ acetosyringone and $25 \mathrm{mM}$ of $\mathrm{MgCl}_{2}$ was added and the mixture and resuspended in $10 \mathrm{mM}$ MES buffer. A small amount of Tween 20 was then added to act as a surfactant, generally $10-50 \mu \mathrm{L}$ 
for every $5 \mathrm{ml}$ will be sufficient. The leaf to be agroinfiltrated was cleaned with cotton soaked in $50 \%$ ethanol and then wiped with distilled water and a small nick was introduced with a razor. The solution containing the MES-Agrobacterium solution was then aspirated into a needleless syringe and the syringe was placed at the nicked underside of the leaf and gentle pressure was applied with a finger providing counterpressure on the other side of the leaf. Done correctly, a damp patch as shown in Figure 2 will seem to spread over the surface of the leaf. This process was continued until all the leaves are agroinfiltrated. It is vital to keep the agroinfiltrated plants in a high moisture area for a few days to counteract the effects of the stress from the agroinfiltrated process or the plants will wilt.

Detection of transformed regions of the plants was carried out through the spraying of 25 mM4-MUG (4-methylumbeliferil- $\beta$-galactopyranoside) mixed with $20-50 \mu \mathrm{L}$ of Tween 20 for every 5 $\mathrm{mL}$. Plants sprayed with 4-MUG solution was incubated at $37^{\circ} \mathrm{C}$ for 30 minutes to 1 hour before screening with a ultraviolet (UV) lamp producing UV rays at $\lambda_{365}$. Transformed regions expressing the gus gene will appear as mild blue spots on a generally reddish background.

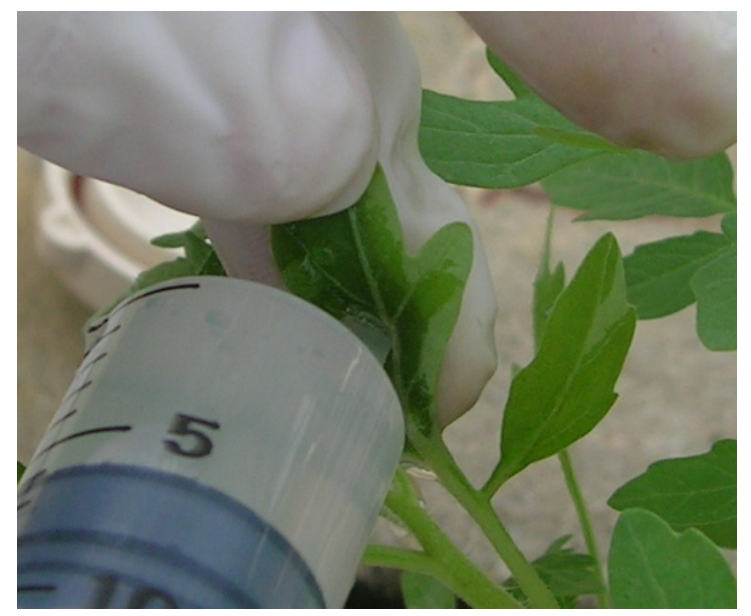

Figure 2. Successful agroinfiltration as indicated by the spreading of the damp spot on the leaf surface which shows that the solution has entered through the stomata.

\section{Vacuum infiltration}

Transformation by vacuum infiltration was also carried out in order to generate transformed plantlets. Newly germinated seedlings were grown on damp cotton until it reached the 2 leaf stage. The seedlings were then placed in MES-Agrobacterium solution with Tween 20 as previously described and was then vacuum infiltrated by applying a vacuum followed by suddenly breaking the vacuum. A nondestructive method for detecting transformed regions of the plants was developed by adding $2 \mathrm{mM} 4-\mathrm{MUG}$ on the agar plate surface and spreading it with a glass 'hockey stick'. Plants were placed directly on the plates so that a large portion touched the media surface and then kept in the media for 3-7 days and finally incubated at $37^{\circ} \mathrm{C}$ for 30 minutes to 1 hour before transferring to a new media without addition of 4-MUG. The spent media containing the 4-MUG was then screened with an ultraviolet (UV) lamp producing UV light at $\lambda_{365}$ to detect for a luminescent signal at transformed regions on plant leaves.

\section{PCR and T-DNA finger printing analysis}

Samples that gave positive GUS response were then tested by PCR for confirmation of gus gene and then T-DNA fingerprinting for copy number detection. Leaf punches were taken from the center of the detected GUS expression.T-DNA fingerprinting (Theuns et al., 2002) was carried by extracting DNA from leaf punches of regions showing expression of the GUS gene. DNA is then digested with MseI and then ligated with a set of adaptors. Amplification was done using a biotin labeled T-DNA insert gene specific promoter and a promoter based on the adaptors. Streptoavidin binding was utilized to isolate any amplified fragments carrying regions of homology to the TDNA specific promoter. Further amplification was done for fragments which have both an adaptor and the $\mathrm{T}$ - 
DNA insert region in order to quantitate the copy number of the T-DNA.

\section{Results and Discussion}

Putative transformed plantlets were embedded on agar plates containing 4MUG and after incubation and transfer of the plantlets. The spent media was scrutinized under UV light in order to detect for bleed over of GUS from transformed regions of the plants into the media plate. Analysis of the plates showed that not only can media containing 4-MUG be used to detect for GUS expression as shown in Figure 3(a), it can also show the localization of the expression as shown in Figure 3(b) when the incubation time was reduced enough to avoid too much build up of GUS. Figure 3(b) showed an example of a plate showing expression along the stem region and also expression from the leaf region, the plant outline was drawn in marker pen and kept track of before transferring into fresh media in order to allow for sample tracking of positive GUS expression and negative GUS expression.

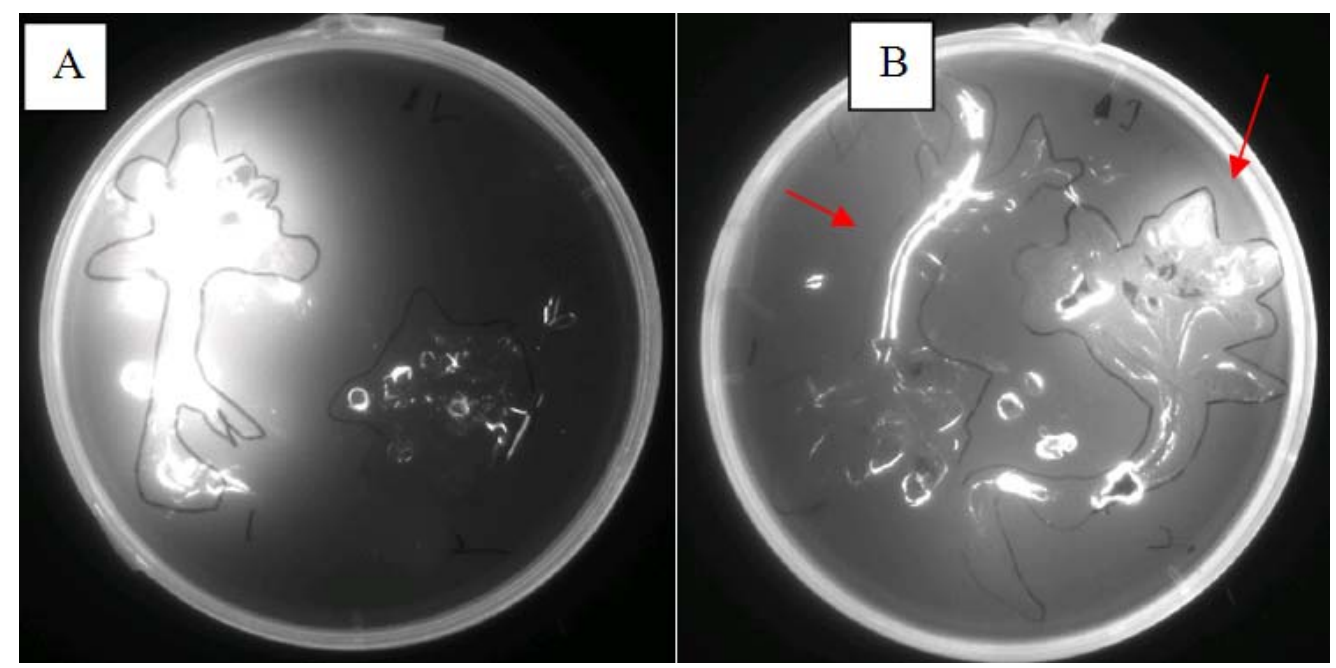

Figure 3. (a) An example of a transformed plant (left) versus a control plant (right) and (b) The effect of reducing the incubation time in order to determine the localization of expression.

Red marks indicated regions of the plant where expression of GUS was detected; a faint outline of the plant was drawn with marker in order to correlate the expression pattern to the plant's anatomy.

Non-destructive GUS assay on agroinfiltrated leaves was done and positive GUS response was detected on some leaves as shown in Figure 4 and Figure 5. It was found that direct agroinfiltration tends to produce isolated single expression at the region of infiltration whereas plants that were infected through vacuum infiltration generally tend to have multiple spots distributed throughout the plant. Agroinfiltration via leaf wounding produced single large spots when viewed under UV while vacuum infiltration produced multiple small GUS expression spots on the leaves.

Even though the Agrobacterium spread throughout the entire leaf when agroinfitrated, expression was detected only near the point of infiltration thus giving rise to the question if there were any factors limiting or effecting the ability of the insertion of the new gene. The major difference between agroinfiltration and 
vacuum infiltration is that in vacuum infiltration, insertion was carried out through multiple points and no wounding was introduced. This suggested that there was a possibility that the wounding response of the plants might have a connection to the expression pattern, for example it was possible that the presence of the wounding response related proteins will somehow effected the expression at the wounding site but restricted gene transfer in other regions.

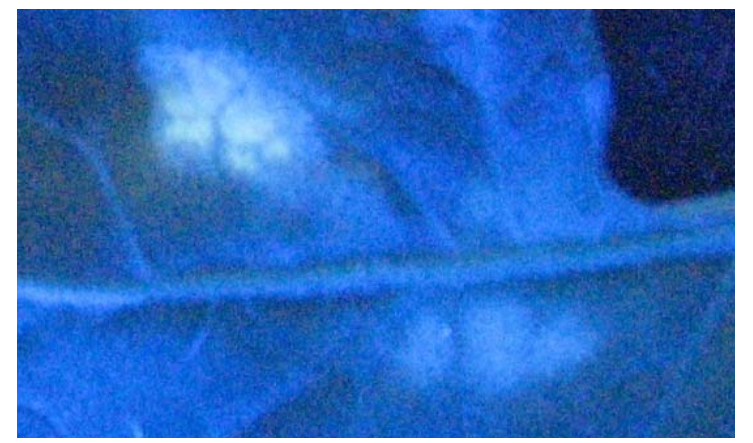

Figure 4. Expression of GUS detected through non-destructive 4-MUG spraying

on leaves transformed via direct leaf agroinfiltration. Bright spots are GUS expression detected via direct MUG spraying on the leaves.
123
4 56
7
$8 \quad 9101112$

Figure 5. Vacuum infiltrated plants with GUS expression. Bright spots are GUS expression detected via direct MUG spraying on the leaves
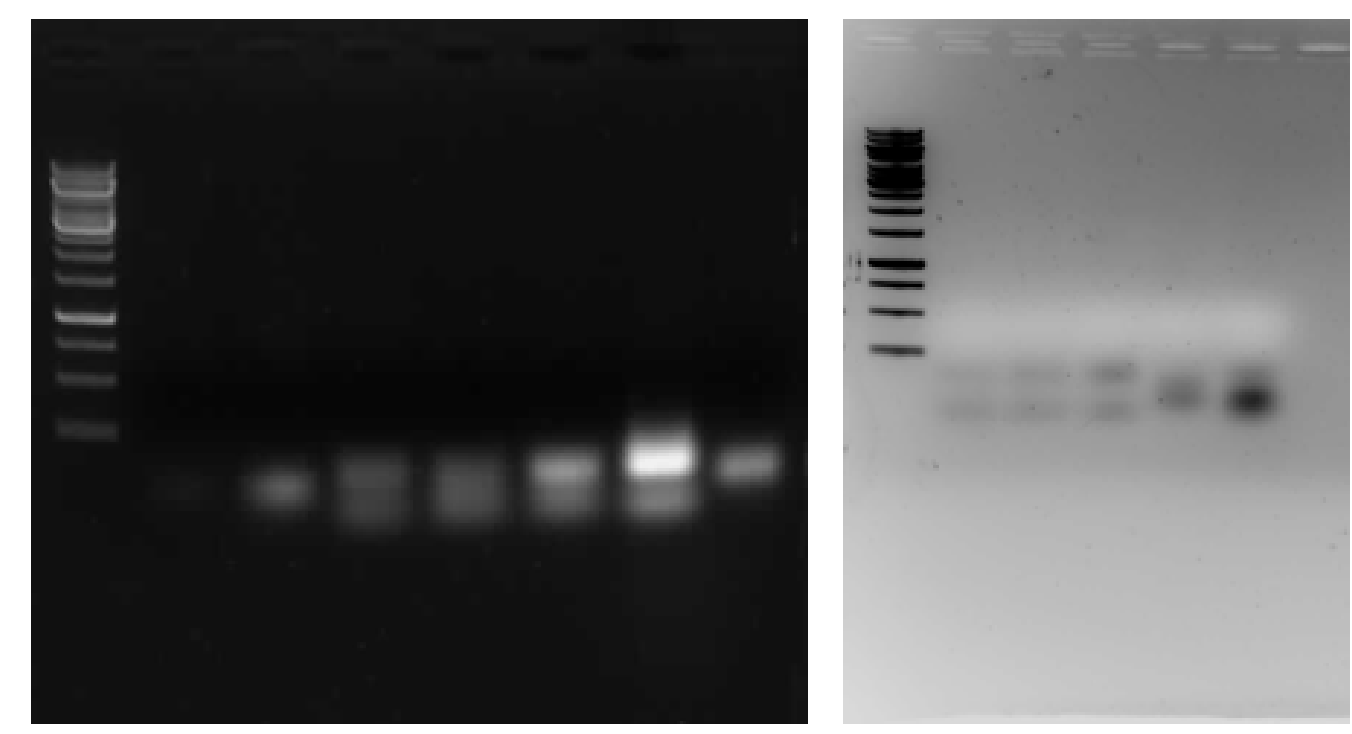

Figure 6. T-DNA fingerprinting results of leaf punches. Lane 1: Negative control; Lane 2-6: TDNA fingerprinting results for samples; Lane7: Control DNA from a known single GUS insertion plant. Lane 8-12: T-DNA fingerprinting results of GUS spots isolated from vacuum infiltration treated plants. 
In order to determine the value of using agroinfiltration or vacuum infiltration for promoter studies, T-DNA fingerprinting was carried out in order to detect the copy number. Single copy numbers are more desirable then multiple copy numbers which will complicate further analysis. Figure 6 showed that the insertion number tends to be low (between 1 and 2) and this method with the T-DNA insertions studies done on other organisms (Meng et al., 2007; Li et al., 2007). The size of the recovered T-DNA tags were short and this indicated that the enzymatic digestion of the genomic DNA should have been made with an enzyme with a lower cutting frequency to obtain longer fragments which can be sequenced to gain more information. This experiment was done in order to test the feasibility of generating a high number of leaf disc transformants and treating each of them as a single transformation event for analysis. The non-destructive method of screening for GUS expression allows us to screen the transformed region multiple times at different times in order to identify insertion of the T-DNA into temporally regulated regions. We believe that this method can be used to take advantage of the chimeric nature of direct agroinfiltration to generate a large amount of transformed materials tagging for regulatory regions. With the advent of single molecule sequencing technology, it is possible to use this method to generate a large amount of transformants which exhibits a single copy number and is linked to the expression of a promoter and sequence them to elucidate the regulatory regions.

Knowledge of the sequence of the promoter region can then be used together with bioinformatics predictions and in planta characterization in order to understand the sequence motives that regulate and characterize a promoter. This knowledge will increase our understanding of how promoters work and may point out to novel sequence motives which can be valuable for deciphering the link between genetic sequences of a promoter and the function of the promoter as well as providing new promoters for plant transformation. The method described in this paper provides an alternative for the current method of generating promoter trapping lines or whole genome sequencing for the purpose of elucidating the sequence and function of promoters and is capable of producing multiple $\mathrm{T}$ DNA tagged cells for research at a relatively low cost, complexity and time.

\section{Acknowledgement}

This work was supported by Ministry of Higher Education of Malaysia through Science Fund grant awarded to Ismanizan Ismail. First author Dinesh A/L Natorajan was a recipient of the National Science Foundation (NSF) fellowship granted by Ministry of Science, Technology and Innovation of Malaysia.

\section{References}

Abuin, A., G. Hansen and B. Zambrovickz. 2007. Gene Trap Mutagenesis. Handbook of Exp. Pharmacol. 178:129-147.

Anuradha, T. S., S. K. Jami, R. S. Datla and P. B. Kirti. 2006. Genetic transformation of peanut (Arachis hypogaea L.) using cotyledonary node as explant and a promoterlessgus: nptII fusion gene based vector; J. Biosci. 31:235-246.

Bouche, N and D. Bouchez. 2001. Arabidopsis gene knockout: phenotypes wanted. Curr. Opin. Plant Biol. 4:111-117.

Galbiati, M., M. A. Moreno, G. Nadzan, M. Zourelidou and S. L. Dellaporta. 2000. Large-scale T-DNA mutagenesis in Arabidopsis for functional genomic analysis. Funct. Integ. Genom. 1:25-34. 
Hsing Y. I., C. G. Chern, M. J. Fan, P. C. Lu, K. T. Chen, S. F. Lo, P. K. Sun, S. L. Ho, K. W. Lee, Y. C. Wang, W. L. Huang, S. S. Ko, S. Chen, J. L. Chen, C. I. Chung, Y. C. Lin, A. L. Hour, Y. W. Wang, Y. C. Chang, M. W. Tsai, Y. S, Lin, Y. C. Chen, H. M. Yen, C. P. Li, C. K. Wey, C. S Tseng, M. H. Lai., S. C. Huang, L. J. Chen and S. M. Yu. 2007. A rice gene activation/knockout mutant resource for high throughput functional genomics. Plant Mol. Biol. 63:351-364.

Li G., Z. Zhou, G. Liu, F. Zheng and C. He. 2007. Characterization of TDNA insertion patterns in the genome of rice blast fungus Magnaporthe oryzae. Curr. Genet. 51:233-243.

Meng Y., G. Patel, M. Heist, M. F. Betts, S. L. Tucker, N. Galadima, N. M. Donofrio, D. Brown, T. K. Mitchell, L. Li , J. R. Xu, M. Orbach, M. Thon, R. A. Dean and M. L. Farman. 2007. A systematic analysis of T-DNA insertion events in Magnaporthe oryzae. Fungal Genet Biol. 44(10):1050-64.

Parinov, S. and V. Sundaresan. 2000. Functional genomics in Arabidopsis: large scale insertional mutagenesis complements the genome sequencing project. Curr. Opin. Biotech. 11:157161.

Serge R., E. Thiry, B. Coemans, S. Windelinckx, R. Swennen, and L. Sági. 2005. Improved T-DNA vector for tagging plant promoters via highthroughput luciferase screening BioTech. 38:763-770.

Sessions A., E. Burke, G. Presting, G. Aux, J. McElver, D. Patton, B. Dietrich, P. Ho, J. Bacwaden and C. Ko. 2002. A high-throughput Arabidopsis reverse genetics system. Plant Cell 14:2985-2994.
Sreenivasan, R. and D. Saha. 2009. Promoter trapping in plants using TDNA mutagenesis. Mol. Tech. Crop Impr. 4:545-577.

Sundaresan V., P. Springer, P. Volpe, S. Haward, C. Dean, J. D. G Jones, H. $\mathrm{Ma}$ and R. Martienssen. 1995. Patterns of gene action in plant development revealed by enchancer trap and gene trap transposable elements. Genes Dev. 9:1797-1810.

Szabados L., I. Kovacs, A. Oberschall, E. Abraham, I. Kerekes, L. Zsigmond, R. Nagy , M. Alvarado, I. Krasovskaja and M. Gal. 2002. Distribution of 1000 sequenced TDNA tags in the Arabidopsis genome. Plant J. 32:233-242.

Theuns I., P. Windels, S. De Buck, A. Depicker, E. Van Bockstaele and M. De Loose. 2002. Identification and characterization of T-DNA inserts by T-DNA fingerprinting. Euphytica 123:75-84.

Van der Hoorn R., F. Laurent, R. Roth and P. J. G. M. De Wit. 2000. Agroinfiltration is a versatile tool that facilitates comparative analyses of Avr9/Cf-9-induced and Avr4/Cf-4induced necrosis. Mol. PlantPathogen Inter. 13:439-446.

Walden R. 2002 T-DNA tagging in a genomics era. Crit. Rev. Plant Sci. 21:143-165.

Yang, Y., H. Peng, H. Huang, J. Wu, S. Jia, D. Huang and T. Lu. 2004. Large-scale production of enhancer trapping lines for rice functional genomics. Plant Sci. 167:281-288. 